is not characteristic of any highly skilled work, and least of all of work having a strong 'mental' component. The operations involved here are marked by complex, co-ordinated and accurately timed activities. The stimuli in response to which these activities are set up are neither simple nor do they usually fall into an order of fixed succession. They hav e the character of a field, or a pattern, which has beco me very highly organized, and may retain its identity in spite of a great diversity of internal arran gement.

It is possible to develop fully controlled experiment lsituations in which these realistic consideration shave full play. When this is done, the picture of fatigue following highly skilled work which emerges has certain strongly marked characters.

In such fatigue the 'standards' accepted and followed by the central nervous system unwittingly deteriorate. The operator tends to think that he is doing better work, because errors treated as significant all the time get wider and wider limits. Until a stage of great fatigue is reached, it is far more likely that the right actions will be performed at the wrong times than that the wrong actions will be performed. If accurate timing is insisted upon, gross mistakes of action may appear. The stimulus field splits up. Its pattern character alters. It becomes a collection of unconnected signals for action, with some of these predominant over all the others. Particu,larly stimuli which are in the margin of the pattern, not closely organized with the central field, are ignored, 'forgotten', and serious lapses of specific reactions occur. There is a marked change in the effect of certain 'distracting', or additional stimuli. Sensations of bodily origin, in particular, become more pressing and insistent, and affect the performance in ways peculiar to the tired operator.

Side by side with all these changes go constant subjective symptoms. Verbal reports about any circumstances connected with known failure of performance become increasingly inaccurate, and errors are regularly projected upon objective conditions, or attributed to the interference of other people. There is a tremendous growth of irritability.

The light thrown by this picture upon the relation of high-level central nervous functions to simpler neuro-muscular mechanisms was dis. cussed.

\title{
ROLE OF RADIATION MUTATIONS IN MANKIND*
}

\section{By Dr. H. J, Muller}

\begin{abstract}
$\mathrm{A}^{\mathrm{c}}$ CCEPTING, on the basis of work with organisms in general, including mice, the conclusion that $\mathrm{X}$-rays and other high-energy radiations produce gene mutations in any human germ cells that are exposed to them, with a frequency exactly proportional to the dose of radiation used, we may ask : What are the conditions governing the manifestation of these mutations ? When attention is confined to the recessive gene-mutations, which appear as the most pronounced and constant, yet insidious, genetic effects, it may be approximately calculated how long the latent period would be between the time of exposure of an individual who afterwards reproduces and the appearance of some descendant manifesting a recessive mutation that had been produced in one of the former's germ cells.
\end{abstract}

For the manifestation of a recessive gene two germ cells having the same allelically acting recessive genes must come together, forming a homozygotean event that would happen with almost negligible frequency in the first-generation offspring, even from two irradiated parents. There are, however, two ways in which a homozygote manifesting the mutated gene could eventually be formed. One is by a germ cell containing it meeting in fertilization another germ cell which chances to contain an identical or allelic recessive gene which, having originated by some independent mutation, was already present in the population in some parallel line of descent. The chance of this event depends upon the frequency of such recessive mutated genes in the general population, and this can be calculated if we know the socalled 'spontaneous' mutation frequency, the rate at which individuals manifesting such genes are being eliminated in competition with normals, and the amount of inbreeding in the population. Sufficient data exist for calculating that under the condition

* Substance of a paper read at the annual meeting during April 28-29 of the U.S. National Academy of Sciences. of random breeding-which would give the highest frequency of such genes-very fow recessive genes the manifestation of which would be of seriously detrimental character would exist in frequencies exceeding 1 in 30 or, more probably, 100 germ cells, in the general population. Thus it would on the average require some $30 \mathrm{or}$, more likely, 100 or more generations of breeding-some $750-3,000$ years of human history-before the mutated gene would manifest itself by this process.

The other way in which the gene could manifest itself would be by a germ cell containing it meeting another containing a relative, so to speak, of the same gene, that is, an identical gene derived from the same ancestral mutated gene of the original treated individual. This requires the breeding together of more or less related individuals- 'inbreeding'-and the chance of its occurrence depends on the total amount of inbreeding occurring in the population. In determining the frequency of this effect a special method of calculation has been employed, utilizing a simple proportionate relation of 1 to 4 which I have calculated to hold between the frequency of two related genes thus meeting each other and the frequency of two individuals having the same name by reason of some near or distant relationship marrying each other. Reckoning from known statistics on the latter events, it can be shown that, even in rural English communities, the chance of a mutated gene manifesting itself by this process is only about 1 in 200 . That is, it would on the average take about 200 generations, or at least 5,000 years, for the gene to attain manifestation in such manner. Both causes operating together would not suffice to give a period of less than 600 years, and more probably one of thousands of years.

Of course these periods are only averages for the time of first manifestation; actually, the first manifestations of the different mutated genes would be 
scattered rather evenly over the entire time from the generation after treatment until two or three times the length of the 'latent period' had elapsed It is to be noted, further, that this protracted 'latent period' does not mean that the mutated genes would tend, collectively, to become lost in the interim, since for each gene that died out by accident, in its 'covered' state, another would, on the average, increase in numbers, and so the final number of individuals first manifesting the mutations would fairly accurately represent the number of individuals produced in the first generation after treatment who had received the newly mutated genes. That is, the damage would be long postponed, but not prevented.

In so far as the individuals manifesting the abnormalities might themselves reproduce they would give them a chance to reappear repeatedly, until competition with the normals finally eliminated them. The recognition of these abnormals as having been produced by the original treatment would, however, be impossible, not only because of the long latent period but because they would appear merely as one relatively small part of the vast number of mutations that had occurred in the distant past, from all causes combined, and that had accumulated in the germ plasm. It can be shown that even if the muta. tion frequency of the population as a whole were doubled, in a given generation, by treatment of all of them with an average dose of, say, $300 \mathrm{r}$. the frequency of mutations that manifested themselves in the population would not be increased, in any one generation, by as much as 1 per cent of their own total, since the manifestation of the new additions to the store of mutations would be scattered over so many generations. If, however, the exposure that doubled the mutation frequency were to be continued generation after generation, the manifestation frequency would gradually rise and finally (after some hundred generations) attain the double value representing the double mutation frequency.

In these circumstances, the advisability of allowing the gonads of a person of reproductive or pre-reproductive age to be exposed to radiation must be decided by balancing the chance of benefit to him against the chance of detriment to future generations. Far more quantitative research is needed on this matter, but it will often be clear that the decision should be in favour of the exposure. Whenever possible, however, a person's gonads should be protected by a lead shield.

As for the question of the mutational effects of natural radiation, while this has been calculated to be of no consequence in flies, the 700-fold greater length of the human generation gives a chance for the accumulation of a sufficient dosage-some 10-15 r. - to have an appreciable effect in view of its also being continued generation after generation. More exact studies are therefore called for concerning the circumstances attending exposure of populations to unusually high amounts of such radiation, as well as of radiation of artificial origin.

\section{SKIN EFFECT IN COMPOSITE AND FERRO- MAGNETIC WIRES}

\begin{abstract}
7 HE British Electrical and Allied Industries
Research Association has published a report by G. G. Sutton on the high-frequency properties of wire specimens*. Novel results have been found in connexion with the effect of a longitudinal magnetic field on wires made of magnetic material. Ferromagnetic wires are shown to have very high 'skin' factors, but the effective permeability determined from the experimental results is shown not to be constant but decreases to a small value as the frequency increases.

Earlier mathematical calculations have assumed a uniform known permeability, but it is known that with ferro-magnetic conductors the permeability varies with the magnetizing force, and hence it must vary over the cross-section of the conductor. It also varies with the current density and the temperature. In addition, hysteresis losses must be considered. Accordingly a mathematical solution is intrinsically very difficult, and it therefore seems necessary to have some direct means of measuring skin factor for magnetic and composite conductors.
\end{abstract}

The author describes two methods of doing this, both methods being based on thermal measurements. The first apparatus consists of two parallel brass tubes inside which were the specimen wire and a standard wire of eureka respectively. The heating effect of the current in the two wires was measured by observing the extensions by means of a travelling

* Technical Report, Reference M/T 69: The High Frequency Properties of various Forms of Wire Specimens. By G. G. Sutton. Pp. $19+13$ plates. (London: British Electrical and Allied Industries Research Association, 1941.) $6 s$. microscope. Both the eureka and iron wires were calibrated with direct current. A simple mathematical relation is then deduced to give the required skin factor. A more accurate method was afterwards developed, mercury being used at first as the indicating fluid, but it was abandoned as it was not sensitive enough. Finally, the liquid decided on was nitrobenzene as its physical properties were found most satisfactory.

The method of measurement developed for skinfactor measurements is shown to give consistent results with different types of specimen. The calculation of results in the case of ferro-magnetic materials is difficult, and in the case of composite materials it is very difficult. The author's method of measurement is direct and calculation is much simplified.

The method can be easily applied to the measurement of high-frequency currents. By further development it would be possible to eliminate many of the difficulties met with in this research. The effect of a longitudinal direct-current field on the highfrequency resistance of a mumetal specimen has been demonstrated; this is in accord with results already published on the are impedance of specimens of mumetal wire at telephonic frequencies. From the results obtained in the ease of ferro-magnetic ma. terials, the effective permeability has been calculated; this tends to a small value as the frequency increases, as indicated by Arkadiew and others. Constructional diagrams are given for making the apparatus, and experimental diagrams are given showing the variation of skin factor with applied D.C. field in the case of a specimen of 26 s.w.G. mumetal wire. 